\title{
Análise ergonômica do atendimento clínico odontológico
}

\author{
Tânia Adas Saliba*, Ana Carolina Bernardes Machado**, Artênio José Ísper Garbin***, Luis Fernando \\ Dahmer Peruchini****, Cléa Adas Saliba Garbin******
}

* Professora, Programa de Pós-graduação em Odontologia Preventiva e Social da Faculdade de Odontologia de Araçatuba, São Paulo, Brasil

** Mestranda em Odontologia Preventiva e Social da Faculdade de Odontologia de Araçatuba, São Paulo, Brasil.

*** Professor Adjunto, Programa de Pós-graduação em Odontologia Preventiva e Social da Faculdade de Odontologia de Araçatuba, São Paulo, Brasil.

**** Mestre em Odontologia Preventiva e Social pela Faculdade de Odontologia de Araçatuba, São Paulo, Brasil

***** Professora Titular, Programa de Pós-graduação em Odontologia Preventiva e Social da Faculdade de Odontologia de Araçatuba, São Paulo, Brasil.

\section{RESUMO}

O presente estudo teve por objetivo analisar aspectos ergonômicos de atendimentos clínicos realizados em quatro especialidades odontológicas. Trata-se de um estudo observacional, para o qual foram filmados 48 procedimentos odontológicos, das especialidades de Endodontia, Cirurgia, Dentística Restauradora e Periodontia, realizados por acadêmicos concluintes de um curso de Odontologia em um laboratório de ergonomia. A postura ergonômica foi analisada por meio de um instrumento baseado nas especificações da ISO/FDI (International Standards Organization e a Federation Dentaire Internacionale). De acordo com essa classificação, são observados oito aspectos durante o atendimento, que envolvem o profissional e o equipamento utilizado, recebendo um ponto para cada item corretamente executado. Os tratamentos de Endodontia tiveram média de 3,69 pontos positivos; Cirurgia 4,03; Dentística Restauradora 4,26 e Periodontia 5,99 pontos. O tempo médio de atendimento foi de 62,3 minutos, sendo que os de maior duração resultaram em número maior de infrações ergonômicas $(\mathrm{p}<0,01)$. Quanto maior a complexidade do procedimento realizado, menor a adequação aos princípios ergonômicos $(\mathrm{p}=0,01)$. Em conclusão, a postura de trabalho foi insatisfatória principalmente em especialidades que demandaram maior tempo clínico.

Descritores: Engenharia Humana. Postura. Odontologia. Especialidades Odontológicas. 


\section{INTRODUÇÃO}

Os distúrbios musculoesqueléticos são muito comuns em cirurgiões-dentistas, principalmente devido à posição de trabalho. $\mathrm{O}$ posicionamento da cabeça do paciente sempre muito próxima ao operador e a pequena área de trabalho que é a cavidade bucal fazem com que o profissional adote posturas estáticas inflexíveis, laboriosas e prolongadas ${ }^{1,2}$. Como consequência disso, é frequente o aparecimento de lesões de esforço repetitivo ou doenças osteomusculares relacionadas ao trabalho (LER/DORT), derivadas de atividades profissionais em que o esforço repetitivo é uma constante, onde há grande demanda muscular por várias horas diárias. É de extrema importância que o cirurgião-dentista se conscientize dos meios de prevenção dessas patologias visto que apresenta grande susceptibilidade para desenvolver estes tipos de doenças, devendo se atentar aos primeiros sinais e sintomas para que o tratamento seja feito o mais precocemente possível, aumentando assim o tempo de exercício da profissão e a qualidade de vida profissional ${ }^{3,4}$.

Grande parte das vezes os distúrbios osteomusculares passam despercebidos e o profissional acaba se acostumando com a dor ou incômodo, esquecendo-se dos cuidados com a própria saúde. Com a inserção de conteúdos de Ergonomia nas instituições de ensino o profissional, desde a graduação, apresenta a preocupação em adotar posições corretas durante os atendimentos, utilizando equipamentos $\mathrm{e}$ instrumentos que favoreçam o seu trabalho dentro dos princípios ergonômicos ${ }^{5}$.

A ergonomia aplicada à Odontologia tem como propósito reduzir o estresse cognitivo e físico bem como o de prevenir as doenças relacionadas ao exercício da profissão, por meio de adequações do ambiente de trabalho e da otimização do atendimento, eliminando passos desnecessários para a execução dos procedimentos, uma vez que o profissional quando confortável em seu trabalho consegue prevenir distúrbios musculoesqueléticos, melhorar sua qualidade de vida e proporcionar mais conforto ao paciente . $^{6}$

Apesar da existência do ensino de ergonomia nas universidades e do constante apelo da engenharia humana, existe entre os profissionais da Odontologia uma grande prevalência de distúrbios relacionados com a prática da profissão, principalmente dores nas costas e no pescoço ${ }^{7}$. Assim, pode-se ressaltar que fatores como hábitos errôneos, posturas inadequadas, jornadas de trabalho abusivas e a repetição de um mesmo padrão de movimento, resultam em baixa produtividade e acometem gravemente a saúde do cirurgião-dentista, podendo causar limitações permanentes na atividade profissional $^{8}$.

Ainda são escassos os estudos sobre a ergonomia aplicada à Odontologia, especialmente na avaliação e orientação postural de acadêmicos nas clínicas de ensino. Desta forma, foi objetivo deste estudo realizar uma análise observacional da postura ergonômica de acadêmicos de Odontologia durante $\mathrm{o}$ atendimento nas especialidades de Endodontia, Cirurgia, Dentística Restauradora e Periodontia.

\section{METODOLOGIA}

Foi realizado um estudo quanti-qualitativo, observacional e descritivo. A pesquisa foi realizada no Laboratório de Ensaios Ergonômicos da UNESP, São Paulo, Brasil. O laboratório é composto por um consultório odontológico completo, com quatro fontes de captura de vídeo e uma sala de observação anexa.

O consultório possui um equipamento odontológico classificado segundo a ISO/FDI (International Standards Organization / Federation Dentaire Internacionale) $)^{9}$ como $3 / 3$, ou seja, tanto o equipo quanto a unidade auxiliar são semimóveis e encontram-se em movimento sobre a cadeira.

As filmagens foram realizadas por quatro câmeras profissionais CCD color Keep HDL (HDL, Itu, SP, Brasil). O monitoramento do atendimento foi feito com o auxílio de uma placa de captura para quatro canais (Pico 2000, HDL, 
Itu, SP, Brasil), de transmissão digital geovision, que capturou as imagens que posteriormente foram transferidas para o computador. Para a visualização simultânea na tela do computador foram utilizadas quatro fontes $500 \mathrm{Ma} / 12 \mathrm{~V}$ (HDL, Itu, SP, Brasil) ${ }^{10}$.

Participaram deste estudo seis acadêmicos concluintes do curso de Odontologia os quais realizaram 48 procedimentos odontológicos, 12 de cada uma das especialidades em estudo.

Os procedimentos foram padronizados e executados em elementos dentais anteriores: tratamentos endodônticos de dentes com polpa viva ou necrosada, exodontias simples, restaurações com resina composta de classes I, III, ou IV de Black e tratamentos de raspagem, alisamento e polimento radicular. Quando era necessária a realização de mais de um procedimento da mesma especialidade, respeitavase o intervalo de uma semana para evitar o efeito de repetição das ações.

Os atendimentos foram gravados pelas câmeras de monitoramento e as filmagens armazenadas para análise. As avaliações foram realizadas por um examinador calibrado, em diferentes momentos: com o filme parado, iniciando no minuto 1 , com novas paradas $\mathrm{e}$ avaliações após cada 10 minutos de filme. Para realizar a avaliação postural foi utilizado instrumento baseado nas normas preconizadas pela ISO/FDI para os atendimentos clínicos odontológicos, que é composto por 8 aspectos que devem ser seguidos, conforme mostra o quadro 1.

De acordo com a classificação de pontuação de aspectos ergonômicos proposta por Garbin et al. $(2011)^{6}$, a pontuação é classificada como insatisfatória ou satisfatória quando a soma é menor ou maior/igual a quatro, respectivamente. ${ }^{6}$

$\mathrm{O}$ avaliador observava o atendimento do acadêmico, atribuindo pontos aos itens corretamente executados, portanto 8 (oito) pontos positivos eram atribuídos quando todos os itens estavam corretos.

Quadro 1. Descrição dos aspectos observados nos atendimentos segundo o proposto pela ISO/FDI.

\begin{tabular}{|c|c|c|}
\hline Aspecto & Região & Observação \\
\hline 01 & Pernas & $\begin{array}{l}\text { O ângulo formado entre a parte posterior da coxa e a panturrilha deve } \\
\text { ser de cerca de } 110^{\circ} \text { ou um pouco mais, com as pernas levemente } \\
\text { esticadas. }\end{array}$ \\
\hline 02 & Coluna Cervical & $\begin{array}{l}\text { O profissional deve sentar-se simetricamente ereto e tão distante o } \\
\text { quanto for possível no assento. O tronco pode inclinar-se para frente } \\
\text { em no máximo } 10^{\circ} \text {. Deve-se evitar rotações e inclinações laterais. }\end{array}$ \\
\hline 03 & Pescoço & A cabeça do cirurgião-dentista pode inclinar-se em até $25^{\circ}$. \\
\hline 04 & $\begin{array}{l}\text { Membros } \\
\text { Superiores }\end{array}$ & $\begin{array}{l}\text { Os membros superiores devem estar ao lado da parte superior do corpo } \\
\text { na frente do tronco, com o antebraço levantado em aproximadamente } \\
10^{\circ} \text { com um limite máximo de } 25^{\circ} \text {. }\end{array}$ \\
\hline 05 & Pés & $\begin{array}{l}\text { Os pés devem ficar apoiados inteiramente no chão e a unidade do pedal } \\
\text { deve ser posicionada próximo a eles, evitando movimentos laterais. }\end{array}$ \\
\hline 06 & Olhos & $\begin{array}{l}\text { O campo de trabalho (boca do paciente) deve ficar alinhado com a } \\
\text { frente da parte superior do corpo e a distância entre a boca do paciente } \\
\text { e os olhos do operador deve ser de } 35 \text { a } 40 \mathrm{~cm} \text {. }\end{array}$ \\
\hline 07 & $\begin{array}{l}\text { Materiais } \\
\text { utilizados }\end{array}$ & $\begin{array}{l}\text { Os instrumentais devem ficar dentro do campo de visão do profissional } \\
\text { e a uma distância de } 20 \text { a } 25 \mathrm{~cm} \text {. }\end{array}$ \\
\hline 08 & Luz & O feixe de luz deve ser mantido paralelo à direção de observação. \\
\hline
\end{tabular}


Os acadêmicos e os pacientes foram informados sobre as filmagens e a observação do atendimento pelo pesquisador, autorizando por escrito à execução da pesquisa e a possível divulgação das imagens, atendendo aos requisitos para a realização de pesquisas com seres humanos, Resolução No 466, de 12 de dezembro de 2012. A pesquisa foi aprovada pelo Comitê de Ética em Pesquisa da Faculdade de Odontologia do Campus de Araçatuba-UNESP (023/2008).

Os dados foram anotados em uma ficha de avaliação individual do procedimento contendo os dados de cada atendimento e, posteriormente foram transcritos para o programa Epi Info 7.1.5. Foram empregados testes estatísticos descritivos e não paramétricos, ao nível de significância de 5\%.

\section{RESULTADOS}

No total de 2.991 minutos de gravação de atendimentos odontológicos analisados, foram realizadas 274 avaliações ergonômicas: 86 $(31,4 \%)$ referentes aos procedimentos de Endodontia; 72 (26,3\%) de Cirurgia; 64 (23,4\%) nos procedimentos de Dentística Restauradora e $52(19,0 \%)$ na especialidade de Periodontia.

A duração média dos atendimentos foi de 62,3 minutos. Os tratamentos endodônticos duraram em média 76,6 min; os de Cirurgia 64,4 min; de Dentística Restauradora 59,6 min e os de menor duração média foram os de Periodontia, com média de 48,6 min.

A análise realizada durante o atendimento odontológico evidenciou a maior média de posturas de trabalho inadequadas na especialidade de Endodontia, seguida da Cirurgia, Dentística Restauradora e Periodontia (tabela 1).

A tabela 2 mostra a classificação das especialidades estudadas, segundo a pontuação dos aspectos ergonômicos ${ }^{16}$.

A tabela 3 apresenta os resultados do teste de associação entre o tempo de trabalho e a média de pontos positivos observados com relação à ergonomia. O teste exato de Fisher apontou diferença estatisticamente significante entre o tempo de trabalho e média de pontos positivos $(\mathrm{p}<0,01)$.

Tabela 1 - Média, mediana, desvio-padrão, valores mínimo e máximo observados nas médias de pontos positivos durante os atendimentos por especialidade.

\begin{tabular}{lccccc}
\hline & Média & Mediana & Desvio-Padrão & Valor mínimo & Valor máximo \\
\hline Endodontia & 3,69 & 3,8 & 0,56 & 2,1 & 4,3 \\
Cirurgia & 4,03 & 4,1 & 0,34 & 3,5 & 4,5 \\
Dentística & 4,26 & 4,25 & 0,75 & 2,7 & 5,4 \\
Periodontia & 5,99 & 6,15 & 0,83 & 4,3 & 7,3 \\
\hline
\end{tabular}

Tabela 2 - Distribuição numérica e percentual dos atendimentos segundo média de pontos positivos observados durante os atendimentos nas especialidades estudadas.

\begin{tabular}{lllll}
\hline Especialidade & \multicolumn{2}{l}{ Pontuação < 4 (insatisfatória) } & \multicolumn{2}{c}{ Pontuação $\geq$ 4 (satisfatória) } \\
\hline & $\mathrm{n}$ & $\%$ & $\mathrm{~N}$ & $\%$ \\
\cline { 2 - 5 } Endodontia & 7 & 43,7 & 5 & 15,6 \\
Cirurgia & 5 & 31,3 & 7 & 21,9 \\
Dentística Restauradora & 4 & 25,0 & 8 & 25,0 \\
Periodontia & 0 & 0,0 & 12 & 37,5 \\
\hline Total & 16 & 100,0 & 32 & 100,0 \\
\hline
\end{tabular}


Tabela 3 - Relação entre a média de pontos positivos observados nos atendimentos e o tempo de trabalho.

\begin{tabular}{lcccc}
\hline Tempo de trabalho & \multicolumn{2}{c}{ Pontuação $<\mathbf{4}$} & \multicolumn{2}{c}{ Pontuação $\geq \mathbf{4}$} \\
\hline & $\mathrm{n}$ & $\%$ & $\mathrm{n}$ & $\%$ \\
Maior que 60 minutos & 12 & 75,0 & 12 & 37,5 \\
Menor que 60 minutos & 4 & 25,0 & 20 & 62,5 \\
\hline Total & 16 & 100,0 & 32 & 100,0 \\
\hline
\end{tabular}

Teste Exato Fisher $\mathrm{p}<0,01$

Observando-se as filmagens foi possível identificar diversos casos de postura inadequada que podem desencadear distúrbios musculoesqueléticos. Dentre essas posturas, destacam-se flexão, inclinação e rotação da cabeça e do tronco, flexão e adução dos membros superiores, flexão e extensão dos joelhos.

O maior número de posições corporais incorretas foi verificado durante a realização de procedimentos de Endodontia com uma média de 3,69 pontos corretos em oito quesitos avaliados.

Os problemas posturais mais prevalentes observados foram angulação da posição das costas em relação ao encosto, sendo que na grande maioria das vezes esta formava um ângulo superior a $30^{\circ}$; a inclinação e a rotação da cabeça e a elevação dos membros superiores a uma angulação superior a $10^{\circ}$.

Observou-se na especialidade de Cirurgia uma média de 4,03 pontos positivos. A falta de postura adequada do cirurgião-dentista em relação ao equipamento prevaleceu, evidenciando a angulação do tronco com o encosto, o ângulo formado entre a parte posterior da coxa e a panturrilha, a inclinação da cabeça superior a $25^{\circ} \mathrm{e}$ os membros superiores elevados como os mais exacerbados.

Os atendimentos na área de Dentística Restauradora atingiram média de 4,26 pontos positivos. Foi observada a dificuldade dos operadores em manter postura adequada da coluna em relação ao encosto do mocho, bem como inclinação e rotação do pescoço e a posição do pedal em relação ao pé, estando na maioria das vezes um ao lado do outro, fazendo com que o operador necessitasse fazer movimentos de lateralização dos pés e. a posição dos membros superiores com o antebraço elevado em mais de $25^{\circ}$.

Os procedimentos de Periodontia foram os que mostraram os melhores resultados, com uma média de 5,99 pontos positivos. A angulação formada pela perna quando o profissional encontra-se sentado deve estar entre $90^{\circ}$ e $110^{\circ}$, porém observou-se que na maioria dos atendimentos esse ângulo era inferior ao de $90^{\circ}$. A incorreta posição em relação ao encosto do mocho também se mostrou bem prevalente, porém sem a presença de torções do tronco. Observou-se boa movimentação do operador, alterando sua posição dentro do relógio virtual desenhado no chão para melhor atender às necessidades do elemento dental que estava trabalhando.

\section{DISCUSSÃO}

Nesse estudo sobre a aplicação dos princípios ergonômicos na prática de especialidades odontológicas, os procedimentos que demandaram maior tempo para sua execução foram os que apresentaram maior frequência de posturas inadequadas.

Nas últimas décadas houve grande evolução no desenvolvimento de novos materiais e equipamentos odontológicos, porém não tiveram grandes avanços ergonômicos quanto à saúde do trabalhador ${ }^{11-13}$.

A prevalência de distúrbios osteomusculares em cirurgiões-dentistas é elevada. Autores relatam que $55 \%$ dos dentistas entrevistados relataram dor lombar e 38,3\% dores cervicais ${ }^{14}$. Estudos demonstram que cerca da metade dos dentistas relataram algum tipo de problema relacionado com o trabalho, principalmente a dor lombar ${ }^{7}$.

As queixas de dor são muito comuns entre 
cirurgiões-dentistas, pois os profissionais ficam sentados em uma mesma posição por grandes períodos de tempo e na maioria das vezes de forma não ergonômica ${ }^{15,16}$.

A utilização de um sistema de monitoramento e filmagem com o uso de várias câmeras simultâneas permite a avaliação e a adequação da postura de trabalho por parte do profissional, além de contribuir para a elaboração de um consultório ergonomicamente planejado, com os equipamentos racionalmente distribuídos. Esta por sua vez permitirá ao cirurgião-dentista um aumento na sua produtividade, além de melhorar a qualidade do serviço prestado e diminuir a fadiga na equipe de trabalho ${ }^{17}$.

O sistema de avaliação da postura ergonômica e o modelo de disposição e estruturação do consultório odontológico baseado em normas propostas pela parceria entre a ISO e a FDI buscam aperfeiçoar o trabalho do cirurgiãodentista, possibilitando a este possuir mais saúde, conforto e segurança no seu ofício. A ISO e a FDI promoveram uma reestruturação para a padronização do trabalho diário do consultório odontológico estabelecendo normas e diretrizes ergonômicas ${ }^{17}$.

Por meio das proposições do ISO/FDI, montou-se um sistema de avaliação do tipo lista de verificação, para identificação de pontos de risco no trabalho. Este método foi adotado neste estudo por ser especifico para a Odontologia, uma vez que existem diversas formas de se avaliar a postura ergonômica de trabalho, para se determinar o risco de distúrbios osteomusculares ${ }^{18}$. Nesta classificação, o profissional deve estar em uma posição considerada neutra da região da coluna lombar e torácica, podendo haver uma flexão de até $30^{\circ}$ da cervical com os membros superiores, ou seja, braços dobrados ao longo do tronco ${ }^{19}$.

Existe uma relação comprovada entre as posturas estáticas ou com pouco movimento e as desordens musculoesqueléticas em diversas profissões. O exercício da Odontologia é um fator de risco para o desenvolvimento destas desordens $^{20}$. Na Endodontia, o operador se mantém durante longo tempo em posições estáticas durante a limpeza e preparação do canal radicular, este pode ter sido o fato de a especialidade apresentar pontuações menores que a Cirurgia, área que demanda mais força e movimentos.

A Endodontia exige do profissional um alto grau de precisão, o que faz com que este necessite de um maior tempo de trabalho, levando-se ainda em consideração o estágio de aprendizado dos operadores neste estudo, que deve ser observado em todas as especialidades estudadas. Outro fator que acaba gerando problemas posturais do cirurgião-dentista que atua nesta área é o campo de trabalho muito pequeno e de difícil iluminação ${ }^{21}$.

Os acadêmicos, atuando durante os procedimentos endodônticos, realizavam movimentos de torção, tanto do pescoço, quanto das costas, para conseguir uma melhor visualização do reduzido campo de trabalho. Os problemas relacionados com esta falta de visualização ocorrem devido ao local de trabalho ou ainda pela falta de iluminação. A iluminação do campo operatório durante os procedimentos de endodontia estava adequada segundo as normas propostas pelo ISO/FDI, restando apenas o fator relacionado à pequena área de trabalho como causa das torções. Porém, a pequena área de trabalho e a possível falta de iluminação adequada em alguns casos podem levar o profissional a sérios problemas visuais. Na década de $40^{22}$, observou-se que $88 \%$ de 2.400 dentistas questionados afirmaram ter problemas de visão, e mais de $65 \%$ disseram ter dores nas costas. Estas, porém, poderiam ser evitadas com o uso adequado da iluminação e da visão indireta durante o trabalho.

O cirurgião-dentista deve, durante o procedimento odontológico, sentar-se no mocho simetricamente ereto e o mais para trás possível. Esta postura deve permitir que o osso esterno esteja um pouco avançado e levantado, já os músculos abdominais devem estar suavemente comprimidos. As costas ficam apoiadas na parte posterior dos ossos da bacia, isso fará com que ele consiga permanecer em posição ereta ${ }^{23}$. 
Evidencia-se a necessidade de postura correta durante a realização de um tratamento de canal radicular, principalmente em relação ao equipamento odontológico. Neste aspecto, os acadêmicos atenderam aos princípios ergonômicos ao utilizar as ferramentas disponíveis para o sucesso ergonômico durante o tratamento, com exceção dos casos que era exigida o uso da visão indireta $^{24}$.

Alguns autores afirmam que o profissional que deseja melhorar seu desempenho na Endodontia deve adotar posições ergonômicas de trabalho, além de economizar movimentos desnecessários durante o exercício das técnicas ${ }^{24}$.

Nos atendimentos clínicos de Cirurgia houve menor frequência de posturas inadequadas, porém ainda longe do ideal. $\mathrm{O}$ ato de anestesiar o paciente tirou o profissional totalmente da sua postura correta de trabalho. Uma justificativa para esse fato pode estar na tensão exigida para realização desse procedimento. $\mathrm{O}$ medo da anestesia leva o paciente a uma postura de autodefesa, fazendo com que este feche mais a boca e se contraia na cadeira odontológica, dificultando a visão e a ação do profissional ${ }^{25}$.

Observou-se durante os procedimentos a diminuição do ângulo formado entre a parte posterior da coxa e a panturrilha do operador, e uma maior angulação do seu pescoço. A força exercida para a exodontia, fez com que o operador elevasse demasiado seu antebraço, podendo levar a problemas tardios em todos os músculos superiores, bem como dores imediatas devido à sobrecarga ${ }^{17}$.

Para prevenir lesões osteomusculares que foram vistas nos procedimentos de cirurgia, o cirurgião-dentista deve posicionar sua perna formando, entre a parte posterior da coxa e a panturrilha, um ângulo de $110^{\circ}$ ou um pouco mais, mantendo-a levemente esticada. Os membros superiores devem sempre estar ao lado do corpo e levemente à frente do tronco durante o atendimento, esta posição irá minimizar o peso fixo dos ombros e dos membros superiores. Todos os movimentos devem ser minimizados, sendo que os laterais devem ficar entre $15^{\circ}$ e $20^{\circ}$, já os frontais podem se estender até os $25^{\circ}$. Os antebraços podem ser elevados de $10^{\circ}$ a $25^{\circ} 23$.

$\mathrm{Na}$ Dentística Restauradora busca-se a otimização dos movimentos para devolver a correta anatomia aos dentes que estão sendo restaurados, o alto grau de detalhe objetivado pode levar o profissional a buscar posições diferentes para a melhor visualização do local do procedimento, bem como para disponibilização de espaço para diferentes movimentos das mãos, que acabam levando o profissional a adotar posições dolosas para o seu corpo. Estudos definiram o espaço entre 9 e 11 horas como a melhor posição de trabalho para o profissional que está realizando uma restauração. Segundo os autores o que deve ser movimentado de acordo com o local do procedimento é a cabeça do paciente e não o profissional ${ }^{26}$. Neste estudo observou-se que o acadêmico acaba buscando melhores posições, ocasionando torções, e inclinações exageradas da cabeça.

A precisão dos movimentos necessários na realização dos procedimentos de Dentística Restauradora faz com que o operador a eleve em mais de $25^{\circ}$ para frente e para cima seu antebraço. Autores relatam que os efeitos adversos dessa postura podem ser compensados com a diminuição desta angulação e com o apoio da mão direita sobre o polegar esquerdo, enquanto esta faz o afastamento de lábios e bochecha, garantindo assim a precisão necessária ${ }^{26}$.

$\mathrm{O}$ incorreto posicionamento do pedal de acionamento do equipamento odontológico foi um dos mais prevalentes erros ergonômicos observados durante a execução de procedimentos de Dentística Restauradora. O local correto para o pedal é sempre próximo ao pé de acionamento, preferencialmente a frente deste, para que não haja necessidade de movimentar o pé lateralmente durante a operação.

A Periodontia revelou-se como a especialidade em que os acadêmicos trabalharam melhor e com uma maior observação das técnicas ergonômicas. Considerando que os mesmos alunos 
realizaram os procedimentos das diferentes especialidades, pode-se supor que durante o ensino na graduação, a disciplina relacionada enfatizou os aspectos ergonômicos. Foi observado na literatura de periodontia clínica que, aliado aos conhecimentos básicos e específicos da área, havia descrições das posições adequadas para o operador durante a realização de procedimentos de raspagem de cálculo, incluindo a melhor posição por face de cada elemento dental ${ }^{27,28}$.

O posicionamento da fonte de luz mostrouse deficiente nos procedimentos periodontais, onde a iluminação ocorria de forma perpendicular à visão do operador, ao contrário do que é preconizado, onde o feixe de luz deve ficar paralelo à direção de observação, eliminando desta forma as sombras.

Em outro estudo, pesquisadores relatam que um cenário típico pode ser observado nas clínicas das faculdades de odontologia, que é o aluno pegando instrumento após instrumento, dobrando, torcendo e contorcendo seu corpo em uma tentativa de se aproximar cada vez mais do local de tratamento. Esse cenário foi visualizado e filmado durante longos períodos dos atendimentos realizados e podem levar nossos acadêmicos a sérios problemas ocupacionais ${ }^{29}$.

$\mathrm{O}$ acadêmico adota posturas corporais anormais durante o seu trabalho, levando ao aumento do estresse físico e afetando seu desempenho profissional. Estas posturas foram observadas neste trabalho, e elas na maioria das vezes, levavam o acadêmico a ter de desempenhar mais movimentos para poder atingir um objetivo inferior ao que atingiria se estivesse atuando de forma ergonomicamente correta ${ }^{30}$.

É necessário um trabalho conjunto do corpo docente das especialidades no ensino da odontologia, quanto à observação dos princípios da ergonomia. A adoção das posturas adequadas de atendimento pelo acadêmico durante a graduação e pelo profissional é essencial para que este se torne um hábito. $\mathrm{O}$ acadêmico deve ser orientado e estimulado continuamente a empregar os princípios ergonômicos na prática odontológica pois irá replicar o que assimilou e desenvolveu durante o curso de graduação, levando os maus hábitos adquiridos nas clínicas de ensino para o seu dia a dia profissional. Estudos sobre esses aspectos devem ser realizados para minimizar os efeitos deletérios e melhorar a qualidade de vida dos cirurgiões-dentistas.

\section{CONCLUSÃO}

As menores médias de pontos positivos relacionados à postura de trabalho foram observadas nas especialidades de Endodontia e Cirurgia. Posturas inadequadas de trabalho foram observadas nos atendimentos que demandavam maior tempo clinico e precisão pelo profissional.

\section{AGRADECIMENTOS}

Agradecemos ao CNPq (Conselho Nacional de Desenvolvimento Científico e Tecnológico) pela disponibilização da bolsa de mestrado

\section{ABSTRACT \\ Ergonomic analysis of clinical dental care}

This study aimed to analyze ergonomic aspects of clinical care provided in four dental specialties. This is an observational study, which was filmed 48 dental procedures, specialties: endodontics, surgery, restorative dentistry and periodontology, performed by undergraduate dental students in an ergonomics laboratory. The ergonomic posture was analyzed by means of a checklist, based on the specifications of ISO / FDI (International Standards Organization and the Federation Dentaire Internationale), according to this classification are observed eight points during the service, involving the professional and equipment used, getting one point for each item correctly executed. Results: endodontics treatments had an average of 3.69 positive points; surgery 4.03; restorative dentistry 4,26 points and periodontics 5,99 points. The average service time was 62.3 minutes, and the longer duration resulted in a greater number of ergonomic offenses $(p<0.01)$. The greater the complexity of the procedure performed, the less adaptation to ergonomic 
principles $(\mathrm{p}=0.01)$. In conclusion, the working position was unsatisfactory mainly in specialties demanded greater clinical time.

Descriptors: Human Engineering. Posture. Dentistry. Specialties, Dental.

\section{REFERÊNCIAS}

1. Anton D, Rosecrane J, Merlino L, Cook T. Prevalence of musculosketal symptoms and carpal tunnel syndrome among dental hygienists. Am J Ind Med 2002;42(3):248-57.

2. Horton SJ, Johnstone CL, Hutchinson CMW, Taylor PA, Wade KJ. Clinical working postures of bachelor of oral health students. $\mathrm{N}$ Z Dent J 2011;107(3):74-8.

3. Medeiros UV, Segatto GG. Lesões por esforços repetitivos (LER) e distúrbios osteomusculares (Dort) em dentistas. Rev Bras Odontol 2012;69(1):49-54.

4. Abreu MHNG, Costa AR, Braga AR, Moraes GFS. DASH entre estudantes de curso de odontologia, Belo Horizonte, 2007. Rev ABENO 2008;8(1):16-22 .

5. Gouveia LJ, Cymrot R, Smit FLP. Estudo de caso sobre as disfunções ergonômicas em cirurgiões dentistas. In: Proceedings Safety, Health and Environment World Congress; 2010 July 25-28; São Paulo, Brazil. p. 165-9.

6. Garbin AJI, Garbin CAS, Diniz DG, Yarid SD. Dental student's knowledge of ergonomic postural requirements and their application during clinical care. Eur J Dent Educ 2011;15(1):31-5.

7. Moimaz SAS, Saliba NA, Blanco MRB. The women workforce in dentistry in Araçatuba SP. J Appl Oral Sci 2003;11(4):301-5.

8. Silva Jr DS, Schneid JL, Silva DS, Castro AGB, Nunes RD. Distúrbios osteomusculares relacionados ao trabalho em cirurgiõesdentistas: uma revisão de literatura. Rev Amazônia 2013;1(1): 13-18

International Standards Organization. ISO/TC 106/SC 6 Dental equipamento. 2006. Acesso em 20/10/2011. Disponível em: http://www.iso.org/iso/iso_catalogue/catalog e tc/catalogue tc browse.htm?commid=513 20\&published=on\&development $=$ on \&withdr awn $=$ on\&deleted $=$ on

10. Rovida TAS, Garbin AJI, Peruchini LFD, Machado ACB, Moimaz SAS. Ergonomia Odontológica: integrando teoria e prática para o avanço do ensino. Rev ABENO 2015; 15(4):37-44.

11. Ahearn DJ, Sanders MJ, Turcotte C. Ergonomic design for dental offices. Work 2010;35(4):495-503.

12. Schleyer TKL. Why integration is key for dental office technology. J Am Dent Assoc 2004;135 Suppl:4S-9S.

13. Hagan PE, Montgomery JF, O`Reilly JT, editors. Accident prevention for business and industry: engineering and technology. 12th ed. Illinois: National Safety Council; 2001.

14. Ratzon N, Yaros M, Mizlik A, Kanner T. Musculoeskeletal symptoms among dentists in relation to work posture. Work 2000;15(3):153-8.

15. Rucker LM. Technology meets ergonomics in the dental clinic: new toys for old games? J Am Coll Dent 2000;67(2):26-9.

16. Garbin AJI, Garbin CAS, Ferreira NF, Saliba MTA. Ergonomics and the dentist: an evaluation of clinical practice by filming analysis. Rev Odonto Ciênc 2008;23(2):1303.

17. Garbin AIG, Garbin CAS, Diniz DG. Ergonomic standards and guidelines in dentistry: the way for the adopting a healthy sitting working posture. Rev Odontol Univ Cid São Paulo 2009;21(2):155-61.

18. Sarranheira F, Uva AS. Work-related upper limb musculoskeletal disorders risk evaluation: the use of SI and RULA methods. Rev Port Saúde Publica 2006;6:13-36.

19. Valachi B, Valachi K. Preventing musculoskeletal disorders in clinical dentistry: strategies to address the mechanisms leading to musculoskeletal disorders. J Am Dent Assoc 2003; 134(12):1604-12. 
20. Marshall ED, Duncombe LM, Robinson RQ, Kilbreath SL. Musculoskeletal symptoms in New South Wales dentists. Aust Dent J 1997;42(4):240-6.

21. Puentes CG, Saavedra J. Microscopia em endodontia. In: Leonardo MR. Endodontia: tratamento de canais radiculares: princípios técnicos e biológicos. São Paulo: Artes Médicas; 2008. p. 1432-71.

22. Biller FE. The occupational hazards in dental practice. Oral Hyg 1946;36:1194-201.

23. Hokwerda O, Ruijter R, Shaw S. 2006. Adopting a healthy sitting working posture during patient treatment. $1^{\mathrm{a}} \mathrm{ed}$. Groningen.

24. Figlioli MD, Porto FA. Working posture in dentistry: assessment of the positions of the dentist and dental auxiliary. Odontol Mod 1987;14(5):25-37.

25. Singh KA, Moraes ABA, Bovi A. Fear, anxiety and control related to dental treatment. Pesqui Odontol Bras 2000;14(2):131-6.

26. Castro SL, Figlioli MD. Ergonomics applied to Dentistry. Assessment of posture and positions of right-handed work of $\mathrm{CD}$ and assist in dental restorative procedures. J Bras Clin Estet Odontol 1999;3(14):56-62.
27. Carranza Jr FA, Newman MG, Takei HH. Carranza periodontia clínica. 9th ed. Rio de Janeiro: Guanabara Koogan; 2004.

28. Nagle MM, Piveta ACG, Ricci WA, Montandon AAB. Dificuldades relatadas por estudantes de odontologia diante de procedimentos relacionados à periodontia. Rev ABENO 2010;10(1):37-41.

29. Thornton LJ, Barr AE, Stuart-Buttle C, Gaughan JP, Wilson ER, Jackson AD, et al. Perceived musculoskeletal symptoms among dental students in the clinic work environment. Ergonomics 2008;51(4):57386.

30. Wegman JE. The ergonomic posture in a preclinical technique exercise. J Dent Educ 1983;47(10):664-5.

Correspondência para:

Ana Carolina Bernardes Machado

e-mail: anab.odonto@ gmail.com

Rua José Bonifácio, 1193 - Seção de

Pós-Graduação - 16015-050

Vila Mendonça, Araçatuba - SP. 